\title{
Los sistemas mandálicos como herramienta intercultural para organizar la enfermería internacional
}

\section{Mandalicos as a cross-cultural tool systems for organizing the international nursing}

\section{Mandalicos como um sistemas de ferramenta multi-cultural para a organização de enfermagem internacional}

\author{
Pedro Manuel Salas Iglesias \\ Doctor por la Universidad de Alicante, Licenciado Humanidades, Master Oficial Ciencias Enfermería. Enfermero Consejería \\ Salud y Bienestar Social. Centro Personas Mayores La Orden. Huelva \\ Cómo citar este artículo en edición digital: Salas, P.M. (2013) Los sistemas mandálicos como herramienta intercultural para \\ organizar la enfermería internacional.17, 35. Disponible en: http://dx.doi.org/10.7184/cuid.2013.35.11 \\ Correspondencia: Plaza del Generalife, $n^{\circ} 1,5^{\circ} \mathrm{B}, 21002$, Huelva; Correo electrónico: pedrocorreosalas@hotmail.com; \\ Teléf: 639569427. \\ Recibido: 07/01/2013. Aceptado: 24/03/2013.
}

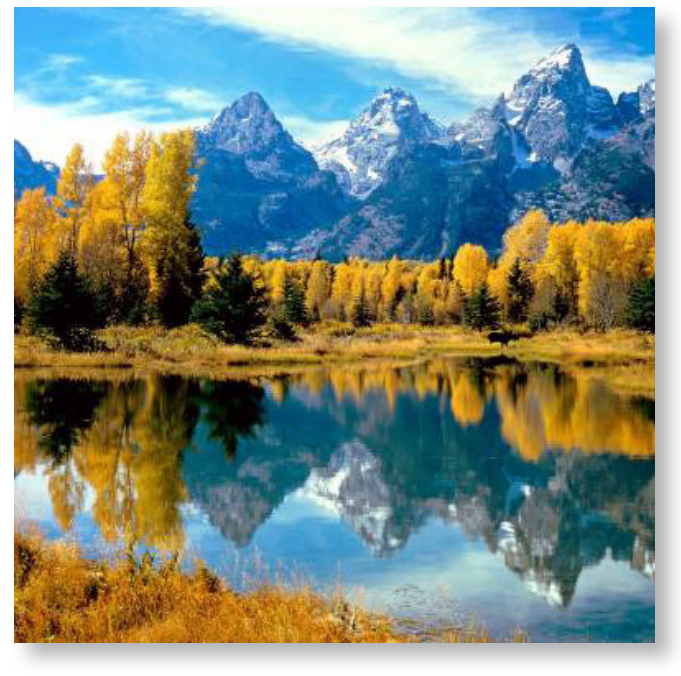

ABSTRACT

It starts from the assumption that the application mandalic model is a useful tool in the organization and delivery of a multicultural care nurses. Throughout history are many examples of mandalas, in psychology and anthropology have also been employed. The letter mandalic organizes cultural diversity from a circle divided into four interrelated subsystems, health is achieved by harmonizing the four subsystems. The circle is constructed from an analysis of the history of life from the expressions, values and needs of the patient / client, besides serving as an organizer of intercultural communication and cultural competence. His way of structuring the environment the person is related to the theory of complex thought of Edgar Morin, and contain many similarities and relationships with other nursing models and theories. It will be used as a tool for self-knowledge and self-transformation, seeking personal excellence, respect for ethnic and cultural variables, considering their individuality with a number of basic needs, spiritual and self-realization. It will serve as an example of creative cultural transformer provided with a value of modern civilization, with a hopeful mindset and transition to a world innovator.

Keywords: Mandálicos cultural systems, cultural care, mandalic letter, cultural competence. 


\section{RESUMO}

Ele parte do pressuposto de que o modelo mandálico aplicativo é uma ferramenta útil na organização e prestação de cuidados de enfermeiros multiculturais. Ao longo da história muitos exemplos de mandalas, em psicologia e antropologia também foram empregadas. $\mathrm{O}$ mandálico carta organiza a diversidade cultural de um círculo dividido em quatro subsistemas inter-relacionados, a saúde é conseguida através da harmonização dos quatro subsistemas. O círculo é construído a partir de uma análise da história de vida das expressões, valores e necessidades do cliente / paciente, além de servir como um organizador de comunicação intercultural e competência cultural. Sua forma de estruturar o ambiente da pessoa está relacionado com a teoria do pensamento complexo de Edgar Morin, e contêm muitas semelhanças e as relações com outros modelos de enfermagem e teorias. Ele será usado como uma ferramenta de auto-conhecimento e auto-transformação, buscando a excelência pessoal, respeito variáveis étnicas e culturais, considerando sua individualidade com uma série de necessidades básicas, espiritual e auto-realização. Ele servirá como um exemplo de transformador cultural criativo fornecido com um valor de civilização moderna, com uma mentalidade de esperança e de transição para um inovador mundo.

Palavras chave: Mandálicos sistemas culturais, cuidado culturais, carta mandálico, competência cultural.

\section{RESUMEN}

Se parte de la hipótesis que la aplicación del modelo mandálico constituye una herramienta útil en la organización y prestación de unos cuidados multiculturales enfermeros. A lo largo de la historia se encuentran multitud de ejem- plos de mandalas, en psicología y antropología también han sido empleados. La carta mandálica organiza la diversidad cultural a partir de un círculo dividido en cuatro subsistemas interrelacionados, la salud se obtiene con la armonización de los cuatro subsistemas. El círculo se construye desde un análisis de la historia de vida a partir de las expresiones, valores y necesidades del paciente/cliente, sirviendo además como elemento de comunicación intercultural y organizador de la competencia cultural. Su forma de estructurar el entorno de la persona guarda relación con la teoría del pensamiento complejo de Edgar Morín, además de contener varias similitudes y relaciones con otros modelos y teorías enfermeras. Será empleado como herramienta de autoconocimiento y autotransformación, de búsqueda de la excelencia personal, de respeto a las variables étnico-culturales, considerando su individualidad con una serie de necesidades básicas, espirituales y de autorrealización. Servirá como ejemplo de creativo cultural dotado de un valor transformador de la civilización actual, con una mentalidad esperanzadora y transición a un mundo renovador.

Palabras clave: Sistemas culturales mandálicos, cuidados culturales, carta mandálica, competencia cultural.

\section{INTRODUCCIÓN}

En este trabajo vamos a investigar la organización internacional de la sociedad actual donde encontramos diferentes culturas en la salud, siguiendo un patrón de referencia mandálico. Para ello nuestra hipótesis tiene como marco sustentador que la aplicación del modelo mandálico constituye una herramienta útil en la organización y prestación de unos cuidados multiculturales enfermeros.

Nos marcaremos como objetivos los siguientes: 
-Referenciar las primeras manifestaciones históricas, culturales y antropológicas del mandala y su asociación con el campo de la salud.

-Señalar las características teóricas del mandala para llevar a cabo su aplicación a los cuidados culturales enfermeros.

-Estudiar la adscripción del mandala en los paradigmas que marca la ciencia y si se adecua a algún modelo teórico del corpus de la enfermería cultural.

-Proponer el modelo mandálico como un modelo válido para su uso por la enfermería como elemento organizador de las culturas de la salud.

- Valorar el manejo del mandala como elemento transformador del entorno de salud pública actual.

\section{MARCO TEÓRICO}

El trabajo de análisis desde una aproximación epistemológica del mandala, proviene de la cosmovisión oriental, del sánscrito, de la cultura hindú, budista, adoptando el significado de dibujo complejo, generalmente circular, que representa las fuerzas que regulan el universo (macrocosmos y microcosmos) y que sirve como apoyo de la meditación (RAE). Desde sus orígenes en tiempos remotos en la India se propagó a otros lugares y culturas orientales e incluso a los aborígenes de Australia.

El hecho de que se encuentren ejemplos de figuras mandálicas en diferentes culturas puede deberse por una parte a la búsqueda de perfección que existe en ellas y que se aprecia en el mandala a través de sus formas concéntricas. A lo largo de la historia se aparición ha sido constante, dotándose el círculo de carácter sagrado en la prehistoria como se puede ver en las primeras construcciones del hombre como en el círculo megalítico de Stonehenge, en el arte cristiano medieval tenemos el ejemplo de la mandorla (almendra), los rosetones de las fachadas y el pavimento con formas de laberintos de las catedrales góticas, las chacanas del pueblo andino, los diagramas de los indios, el símbolo chino del ying yang, el círculo donde se inscribe el hombre de Leonardo Da Vinci, el big-ban, el ojo físico (donde el centro del mandala es la pupila y el iris la expansión de éste), etc. Por tanto podemos consolidar su presencia permanente en diversas culturas y religiones. A este respecto cabe resaltar las palabras del filósofo griego presocrático Empédocles de Agrigento (495-435 a.C) en su visión mandálica de Dios cuando postuló que: "Dios es un círculo cuyo centro está en todas partes y cuya circunferencia no se encuentra en ningún sitio", defendió además la teoría de las cuatro raíces (tierra, agua, aire, fuego donde la salud se obtenía a partir de mantener cierto equilibrio entre éstos elementos).

Si buscamos su aplicación en ciencias como la psicológica, los mandalas significan la totalidad de nuestro ser. Ayudan a curar la fragmentación psíquica y espiritual, expresando la sabiduría interior desde el centro. Viajar a este centro produciría una sensación de calma al reconectar con el ser esencial. La observación contemplativa de su diseño permite una meditación, tomando contacto con lo espiritual al superar las limitaciones presentes y las ataduras del mundo material y conceptual. A este respecto para el psiquiatra Carl Gustav Jung (2002) representa la totalidad de la mente tanto el consciente como el inconsciente, interpretando los sueños a partir de la relación que éstos tienen. Afirmó que el arquetipo de estos dibujos se encuentra firmemente anclado en las expresiones probables del subconsciente colectivo. El centro del mandala es una repre- 


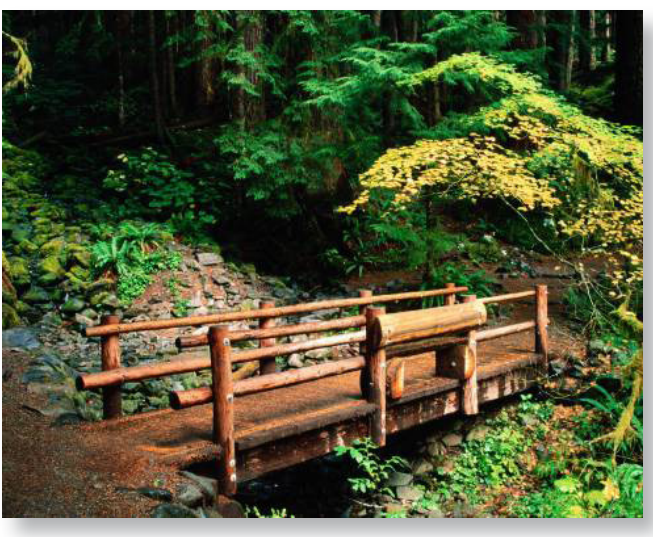

sentación del sí mismo (selbest), intentando lograr la perfección en el proceso de individuación. Su uso tiene un fin de restablecimiento del orden perdido con anterioridad y especialmente con un propósito creador, se genera algo nuevo diferente a lo que existía antes (Ashram, 2012).

Si recurrimos a otra ciencia la antropología, adquiere un sentido expansivo desde el centro a la periferia. El mandala se estructura en una serie de formas geométricas concéntricas organizadas en variados niveles visuales. Los diseños aunque variados mantienen características similares: un centro y puntos cardinales inscritos en círculos y dispuestos con cierta simetría. El centro es el espacio sagrado (centro del universo y soporte de concentración).

Para el antropológo americano Edward T. Hall las culturas están organizadas con ritmos entremezclados comparables al tema de una sinfonía donde están implicados todos los aspectos del complejo comportamiento humano: kinésico (gestualidad), rítmico (temporalidad), proxémico (espacio y cuerpo) y la totalidad de la cultura (lengua, religión, filosofía, etc.) (Fuenmayor, 2012).

Bailey Cunnigham (2003) analiza el mandala como un arte usado en todo el mundo para la autoexpresión, la transformación espiritual y el crecimiento personal, obteniendo una mayor conciencia de cada persona, un símbolo arquetípico en su totalidad. Explorar el mandala nos puede llevar a un viaje a la totalidad, ayudando a la persona a descubrir el centro dentro de sí misma y más allá.

La doctora en antropología social y cultural Alida Carloni emplea el mandala en la explicación metodológica de la diversidad cultural a partir de la Carta Mandálica, compuesta por una serie de partes:

- El centro representa el OM: Buda, Dios.

- Los laterales situados en la parte superior, inferior, derecha e izquierda simboliza los puntos cardinales y estaciones del año, es decir norte/invierno, sur/verano, este/primavera, oeste/otoño.

- Las esquinas constituyen los equinoccios y solsticios y donde se insertan: el fuego/ el verbo, la tierra/el renacimiento, el aire/la transmisión, el agua/la fertilidad.

Además este modelo está dividido metodológicamente en cuatro subsistemas, representados en la figura $1 \mathrm{y}$ tabla 1.

Del análisis derivado de dicho modelo se observa su vinculación con la enfermería humanista y la cultura de los cuidados, donde el hombre equilibrado y sano es el que tiene armonizados los cuatro niveles. La figura circular es abarcadora, integradora y holística, sanadora y considera al paciente/cliente inserto en la naturaleza y en su contexto cultural. Ante la diversidad de culturas de la salud (Spector, 2003) su comportamiento es de ejercer un trato igualitario y situarlas en el mismo nivel de respeto.

Para su empleo desde la óptica enfermera, se realizará un análisis de la historia de vida cultural, construyendo el círculo con cierta habilidad cultural de los diferentes subsistemas mandálicos a partir de las expresiones, valores 
y necesidades del paciente/cliente teniendo en cuenta tanto su historia actual como pasada. La atención de enfermería cultural se complementará con entrevistas y valoraciones posteriores que aumenten los datos sobre las percepciones y la planificación de los cuidados en función de la situación de salud/enfermedad, pudiendo llegar al final a una evaluación cultural.

Por otro lado su empleo puede ser utilizado como base para iniciar los procesos de comunicación intercultural disminuyendo las barreras sociales y culturales entre ambos actores, así como herramienta que permita mejorar el nivel de satisfacción del cliente/paciente con su sistema de atención enfermera/o.

Asimismo se tendrá en cuenta la validación cultural (Alarcón, 2003) que acepta la legitimidad del modelo de salud y enfermedad del paciente considerando su contexto cultural, con actitud comprensiva, de respeto y de integración de aquellos elementos culturales que sean importantes en el proceso de recuperación.

Por otra parte la Carta Mandálica también es un vehículo organizador en el campo de la competencia cultural. Entendida ésta como "la integración compleja de conocimiento, actitudes y habilidades que aumenta la comunicación entre culturas diferentes (cross-cultural comunication) y las interacciones apropiadas/ efectivas con los otros" (Ibarra et al, 2006), Además mantiene su vinculación con los elementos configuradores de dicha competencia como: la conciencia cultural, el conocimiento cultural, la habilidad cultural, el deseo cultural y los encuentros culturales. Si se quiere llegar a ser culturalmente competente se debe tener en cuenta la motivación, propósito y objetivos de ambos actores, una autoevaluación continua, desarrollar las técnicas o habilidades y aproximarnos al conocimiento de los grupos culturales que tratemos (Kersey-Matusiak, 2012).

\section{MATERIAL Y MÉTODOS}

Se ha recurrido a la heurística, análisis de paradigmas y teorías y modelos enfermeros, investigación de enfermería para la evidencia y el empleo del análisis de fuentes documentales y bibliométricas de diferentes bases de datos como: Cuiden, BDEM, Cuidatge, Enfispo, Bilbioteca Cohrane Plus, Medilne Pub Med, Telnet de la Universidad de Sevilla a partir de su motor de búsqueda: Fama. También se han empleado en la investigación varias palabras clave y sus combinaciones booleanas dentro nuestro objeto de estudio como: mandala, carta mandálica, cultural, modelos culturales, cultural and nursing, competencia cultural.

Metodológicamente se han desechado aquellas investigaciones que incorporaban los resultados de las categorías conceptuales en el nombre de autor, agregando las que aparecían en título/resumen. Se trata de un estudio transversal de corte descriptivo basado en el análisis hermenéutico y bibliográfico.

\section{RESULTADOS}

Del análisis del estudio se debe considerar el empleo del mandala en el contexto cultural del cliente/paciente dentro del marco de la competencia cultural. Muchos estudios recurren a la competencia cultural enfermera demostrando que tras un entrenamiento formativo previo pueden mejorar la atención primaria de salud, disminuyendo las disparidades de salud entre niños de padres inmigrantes (Berlin et al, 2010). Por otra parte los cuidados con congruencia cultural adaptada a la minoría étnica que tratemos, disminuye la distancia entre las características de las competencias culturales y la percepción de los clientes de acuerdo a sus necesidades culturales (Costantino et al, 2009, Webb et al, 2010). El empleo de programas culturales teniendo en cuenta sus antecedentes 
culturales mejoran enfermedades relacionadas con el contexto cultural (Choi et al, 2007).

Tras analizar otros modelos y teorías de aplicación en enfermería, encontramos varias similitudes y vinculación de los cuidados mandálicos con ellos, entre los que se destacan:

- El modelo de enfermería del Núcleo, el cuidado y la curación de Hall con los tres círculos entrelazados, representando el círculo del núcleo al paciente, el círculo del cuidado al cuerpo del paciente y el círculo de la curación se refiere a los procesos patológicos o la enfermedad.

- La teoría del logro de metas de King con los sistemas abiertos de los seres humanos en interacción constante con su entorno.

- El modelo de sistemas de Neuman donde la enfermería proporciona una visión multidimensional de la persona como individuo, se contempla como un sistema abierto, dinámico, en interacción constante con el entorno, empleando la enfermera las intervenciones específicas basándose en el grado de reacción al agente estresante.

- El modelo de Adaptación de Roy donde las personas son consideradas como sistemas abiertos cuyas conductas pueden ser clasificadas como respuestas adaptativas o ineficaces y emplean los subsistemas regulador y cognitivo como procesos de control.

- El modelo de la diversidad y la universalidad de los cuidados culturales de Leninger, donde los cuidados culturales pueden ser tanto diversos como universales, su modelo Sunrise sirvió para describir las interrelaciones de la teoría de la diversidad y universalidad de los cuidados culturales, las acciones enfermeras deben ser específicas según la cultura para ser relevantes en la salud y el cuidado del cliente.
- El modelo de competencia cultural de Purnell, formado por doce dominios que se pueden aplicar en el hospital, hogar del paciente, en la comunidad, etc. Promueve la comprensión cultural en períodos de enfermedad, mantenimiento y promoción de la salud, enfocando aspectos emic y etic mediante las opiniones del paciente, familia y comunidad y la forma de valorar las influencias y la identificación con su grupo cultural, su diagrama es un círculo.

Por otro lado, la forma compleja de estructurar el entorno de la persona sana/enferma se encuadra en la teoría del pensamiento complejo de Edgar Morin (Morin, 2012) donde para responder de manera eficiente $y$ sostenible ante las dinámicas socio-culturales, económicas, tecnológicas y políticas que forman parte del mundo globalizado son necesarias unas aptitudes de comprensión, integradoras y coordinadas para que constituyan un todo. Aboga por reformas fundamentales, la educativa, la toma de conciencia ecológica y la política de la civilización proponiendo una acción de conjunto. Enfermería debe adoptar el papel de liberar la fuerza de las buenas voluntades y de acción solidaria, teniendo en cuenta la multidiversidad del mundo real.

\section{DISCUSIÓN Y CONCLUSIONES}

En primer lugar se ha demostrado nuestra hipótesis en el presente estudio. Enfermería empleará los sistemas mandálicos como herramienta para el autoconocimiento y autotransformación de la persona que cuida, así como camino de la búsqueda de la excelencia personal. De la misma manera los utilizará como herramienta organizadora de los elementos que configuran el entorno sociopolítico, económico y de salud que rodea el mundo 
de la persona cuidada. Las variables étnicoculturales y el respeto a la complejidad cultural en el mundo de la salud es una parte esencial a tener en cuenta en las nuevas estrategias internacionales de la salud pública.

Una de las propuestas de las estrategias de futuro de los usos del mandala en salud, consiste en el coloreo y dibujos de éstos. Ayuda a transformar la mente y el entorno al constituir un centro energético reparador, de equilibrio y purificación. En terapias de rehabilitación cognitiva se puede usar esta técnica en enfermedades mentales como el Alzheimer o ante situaciones de estrés emocional o crisis de ansiedad, el colorearlos de adentro hacia afuera posibilita exteriorizar las emociones más fácilmente. Cuando se pinten de afuera a adentro sus efectos se dejarán notar en la búsqueda de un sentido de la vida, expansión de la conciencia y desarrollo de paciencia, con efectos sobre la autoestima y autorrealización. Otros posibles efectos beneficiosos que pueden aparecer son: escuchar la voz de la intuición, aceptándose y queriéndose más, dirigidos hacia una curación física y psíquica.

Desde nuestra profesión trataremos al paciente/cliente inmerso en las diferentes culturas de la salud pero considerando su individualidad, con una serie de necesidades básicas materiales, espirituales y de autorrealización. Los sistemas culturales mandálicos nos servirán como ejemplo de creativo cultural (Ray et al,2000) dotado de un valor transformador de nuestra civilización, sumergida en medio de un cambio de época producido por la gran crisis que nos ha tocado vivir y en un mundo atrapado entre la globalización, las tecnologías de aceleración y un deterioro de la ecología planetaria. Ofertaremos unos cuidados internacionales respetando tanto las normas de cada cultura, los escenarios y los elementos

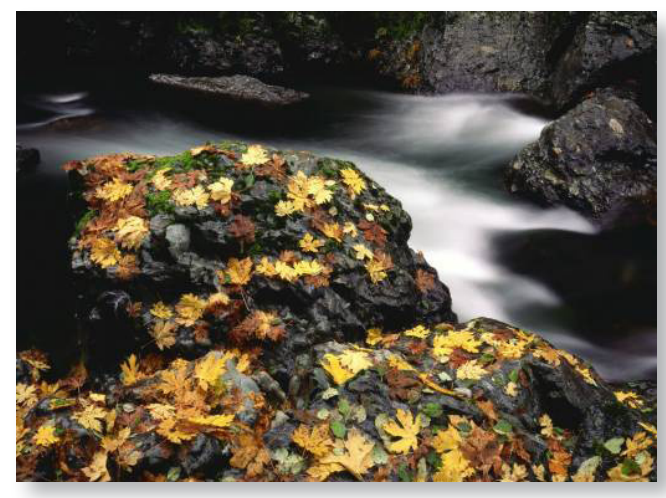

de salud, que respondan a las expectativas del cliente, en un proceso comunicativo y de intercambio, con una mentalidad esperanzadora y de transición hacia un mundo renovador.

\section{BIBLIOGRAFÍAS}

- Alarcón, M. et al (2003) Salud intercultural: elementos para la construcción de sus bases conceptuales. Rev méd. Chile. 131: 1061-1065.

- Ashram, K. (2012) El mandala, disponible en: http://tantriconepali.galeon.com/productos1524072.html. Consultado: $13 / 11 / 2012$.

- Berlin, A. et al (2010) Cultural competence among Swedish child health nurses after specific training: A randomized trial. Nursing \& health sciences.12: 381-91.

- Carloni, A. (2000) Asignatura Antropología Social y Cultural. Facultad Humanidades. Universidad de Huelva, Huelva.

- Costantino, G. et al (2009) Congruence between culturally competent treatment and cultural needs of older Latinos. Journal of consulting and clinical psychology. 77: 941-9.

- Cunningam, B. (2003) Mandala. Viaje a la unidad del ser. Pearson Educación, Madrid.

- Choi, Y.J. et al (2007) Evidence-based nursing: effects of a structured nursing program for the health promotion of Korean women with Hwa-Byung. Archives of psychiatric nursing. 21: 12-6.

- Diccionario Real Academia de la Lengua Española (2012) disponible en: http://lema.rae.es/drae/?val=mandala. Consultado: 10/11/2012, 
- Morín, E. (2012) Edgar Morin. web oficial. Disponible en: http://www.edgarmorin.com/. Consultado: 10/11/2012

- Fuenmayor, V. (2012) Técnicas del cuerpo y técnicas de la danza. Disponible en: http://victorfuenmayorruiz. com/files/tecnicasdelcuerpoytecnicasdeladanza.pdf. Consultado: 14/11/2012

- Ibarra, M. y Siles, J. (2006) Competencia cultural. Una forma de ofrecer cuidados de enfermería. Index enferm 55: 44-48.

- Jung, C. G. (2002) Obra completa. Volumen 9/I. Los arquetipos y lo inconsciente colectivo. Sobre el simbolismo del mándala (1938/1950). En Mándalas. Trotta, Madrid.

- Kersey-Matusiak, G. (2012) Cuidados culturalmente competentes: ¿los tenemos presentes? Nursing. 30: 25-8.

- Purnell, L..A. (1999) El modelo de competencia cultural de Purnell: descripción y uso en la práctica, educación, administración e investigación. Cultura de los Cuidados 3(6): 91-97.
- Ray, P.H. y Anderson, S .R. (2000).The Cultural Creatives: How 50 Million People Are Changing the World. Three Rivers Press. New York, en: Los Creativos culturales un esperanzador descubrimiento sociológico. Disponible en: http://www.sostenibleycreativa.es/cambios-sociales/ los-creativos-culturales/ . Consultado: 06/12/2012.

- Spector, R.E. (2003) Las culturas de la salud. Pearson Educación, Madrid.

- Webb, M.S. et al (2010) Effects of culturally specific cessation messages on theoretical antecedents of behavior among low-income african american smokers. Psychology of addictive behaviors. Journal of the Society of Psychologists in Addictive Behaviors. 24: 333-41.

- Wesley, R.L. (1997)Teorías y modelos de enfermería. McGraw-Hill. Interamericana, México D.F.

Figura 1: Carta Mandálica.

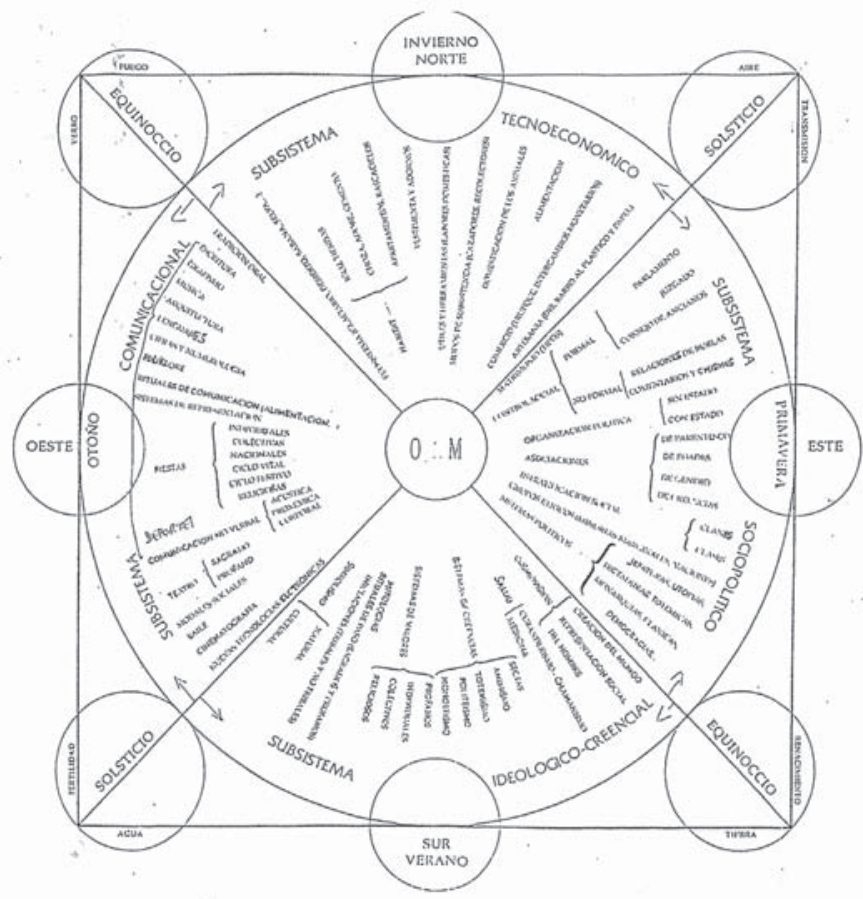

En Carloni, A (2000). Facultad Humanidades. Universidad Huelva. 
Tabla 1: Subsistemas mandálicos

\begin{tabular}{|c|c|c|c|}
\hline Tecnoeconómico & Sociopolítico & Comunicacional & Ideológico-Creencial \\
\hline Ecosistema & Matrimonios & Tradición oral & $\begin{array}{l}\text { Cosmovisión: creación del mundo, } \\
\text { representación social del hombre }\end{array}$ \\
\hline Hábitat & $\begin{array}{l}\text { Control social: } \\
\text { 1.- Formal: parlamento, juzga- } \\
\text { do, consejo ancianos. } \\
\text { 2.- No Formal: relaciones de } \\
\text { burlas, comentarios y chismes }\end{array}$ & Escritura & Salud \\
\hline Útiles y herramientas & Organización políitica & Grafismo & Sistemas de creencias \\
\hline Modos de subsistencia & $\begin{array}{l}\text { Asociaciones: de parentesco, de } \\
\text { edades, de género, de creencias }\end{array}$ & Música & $\begin{array}{l}\text { Sistemas de valores: profanos, } \\
\text { individuales, colectivos, religiosos }\end{array}$ \\
\hline $\begin{array}{l}\text { Domesticación de los } \\
\text { animales }\end{array}$ & Estratificación social & Arquitectura & Mitologías \\
\hline Alimentación & $\begin{array}{l}\text { Grupos étnicos, minorías margi- } \\
\text { nales, nacionales }\end{array}$ & Lenguajes & Rituales de paso \\
\hline Comercio & Sistemas políticos & Cifras y numerología & Iniciaciones: tribales y no tribales \\
\hline \multirow[t]{11}{*}{ Artesanía } & & Folklore & Simbolismo: natural y cultural \\
\hline & & $\begin{array}{l}\text { Rituales de comunicación } \\
\text { (alimentación) }\end{array}$ & \\
\hline & & Sistemas de representación & \\
\hline & & Fiestas & \\
\hline & & Deportes & \\
\hline & & Comunicación no verbal & \\
\hline & & Textos: sagrado, profano & \\
\hline & & Habilidades sociales & \\
\hline & & Baile & \\
\hline & & Cinematografía & \\
\hline & & $\begin{array}{l}\text { Nuevas tecnologías electró- } \\
\text { nicas }\end{array}$ & \\
\hline
\end{tabular}

